\title{
Novel Analysis of Glycan Structures: Nanoscale Approach
}

\author{
Tomas Bertok ${ }^{1}$, Dominika Pihikova ${ }^{1}$, Alena Holazova ${ }^{1}$, Andras Hushegyi ${ }^{1}$, Ludmila Klukova ${ }^{1}$, Jaroslav \\ Filip $^{1}$, Stefan Belicky ${ }^{1}$, Erika Dosekova ${ }^{1}$, Peter Kasak ${ }^{2}$, Jan Tkac ${ }^{1}$ \\ ${ }^{1}$ Institute of Chemistry, Slovak Academy of Sciences \\ Dubravska cesta 9, Bratislava 845 38, Slovakia \\ chemtobe@savba.sk; jan.tkac@savba.sk \\ ${ }^{2}$ Center for Advanced Materials, Qatar University \\ Doha 2713, Qatar \\ peter.kasak@qu.edu.qa
}

\section{Extended Abstract}

Glycans are complex saccharide moieties covering all cell surfaces - presented on different biomolecules. Almost 75\% of all proteins are glycosylated, and these glycans can form thousands of different structures.[1] Moreover, these structures may slightly change during a specific disease progress - depending on the biomarker observed, there is a possibility to distinguish between healthy individuals and people suffering from a specific disease, mostly cancer (prostate cancer using PSA as a biomarker)[2] or autoimmune diseases (rheumatoid arthritis, system sclerosis observing IgG $N$-glycosylation).[3] Viral adhesion on cell surface and subsequent penetration is also dependent on the glycan epitopes present on a cell surface.[4, 5] Using nanoscale manipulation of biorecognition elements (antibodies, lectins as glycan-bindnig proteins or glycans) using self-assembled monolayers (SAMs) allowed to prepare highly sensitive, reproducible and robust biosensors for detection of various analytes - from glycoproteins and whole viral particles to intact cells. Moreover, using SAMs allows to control a biorecognition element's density, orientation and anti-fouling properties of our surfaces.[6] Using nanomaterials like gold nanoparticles of different size or graphene oxide flakes leads to improved characteristics of prepared devices electrochemical and impedimetric biosensors in this case. Electrochemical devices, mainly in combination with different nanostructures, provide cheap, highly reliable and sensitive platform for glycomic analyses.[7]

We present here a novel approach for a glycoprofilation of various analytes (antibodies, PSA, viral hemagglutinins and viruses and eukaryotic cell lines) using small, low cost, highly sensitive electrochemical devices based on different platforms compared to standardly used LC, CE or MS methods for the glycan analysis. Beside electrochemical impedance spectroscopy and voltammetry, other methods for the surface characterization were used (quartz crystal microbalance, surface plasmon resonance, atomic force and scanning electron microscopy and x-ray photoelectron spectroscopy) and our results were compared to outputs from other analytical methods (protein microarray, enzyme-linked lectin assay and MALDI-TOF MS).

\section{References}

[1] J. Tkac, T. Bertok, J. Nahalka, and P. Gemeiner, "Perspectives in Glycomics and Lectin Engineering," in Lectins. vol. 1200, J. Hirabayashi, Ed., ed: Springer New York, pp. 421-445, 2014.

[2] D. Pihikova, S. Belicky, P. Kasak, T. Bertok, and J. Tkac, "Sensitive detection of a prostate specific antigen (PSA) using impedimetric assays with in-situ PSA?s glycan analysis," Analyst, 2015.

[3] E. Paleček, J. Tkáč, M. Bartošík, T. Bertók, V. Ostatná, and J. Paleček, "Electrochemistry of Nonconjugated Proteins and Glycoproteins. Toward Sensors for Biomedicine and Glycomics," Chemical Reviews, vol. 115, pp. 2045-2108, 2015.

[4] A. Hushegyi and J. Tkac, "Are glycan biosensors an alternative to glycan microarrays?," Analytical Methods, vol. 6, pp. 6610-6620, 2014.

[5] A. Hushegyi, T. Bertok, P. Damborsky, J. Katrlik, and J. Tkac, "An ultrasensitive impedimetric glycan biosensor with controlled glycan density for detection of lectins and influenza hemagglutinins," Chemical Communications, vol. 51, pp. 7474-7477, 2015. 
[6] T. Bertok, A. Šedivá, J. Filip, M. Ilcikova, P. Kasak, D. Velic, et al., "Carboxybetaine Modified Interface for Electrochemical Glycoprofiling of Antibodies Isolated from Human Serum," Langmuir, vol. 31, pp. 7148-7157, 2015.

[7] T. Bertok, L. Klukova, A. Sediva, P. Kasák, V. Semak, M. Micusik, et al., "Ultrasensitive Impedimetric Lectin Biosensors with Efficient Antifouling Properties Applied in Glycoprofiling of Human Serum Samples," Analytical Chemistry, vol. 85, pp. 7324-7332, 2013. 